\title{
Vom Leben im Überfluss und seinen Gefahren
}

Die Evolution hat uns Menschen sehr gut ausgestattet, um Zeiten der Dürre und des Nahrungsmangels gesund zu überstehen. Noch nie gab es für die Menschen dagegen eine so lange Periode von Nahrungsüberangebot wie derzeit in den westlichen Industrieländern. Die Biologie unseres Körpers verfügt kaum über Mechanismen, mit ständigem Nahrungsüberfluss umzugehen. Dies könnte dem in der gesamten Gesellschaft verbreiteten Schlankheitsideal einen gesundheitserhaltenden Sinn geben. Aus anthropologisch-evolutionärer Sicht ist anzunehmen, dass Übergewicht in westlichen Industrieländern bei dem bestehenden Nahrungsüberfluss noch weit verbreiteter wäre, wenn keine Gegenregulation mit Breitenwirkung bestünde. Nach einer anthropologischen Theorie [Brown und Konner, 1987] kann durch die gegenregulierende Kraft des Schlankheitsideals auf soziokultureller Ebene die Häufigkeit von schwerer Adipositas und deren Folgeerkrankungen und Risiken (Hypertension, kardiovaskuläre Erkrankungen, Diabetes, bestimmte Krebsfomen) vermindert werden. Essstörungen wie Magersucht und Bulimie wären aus dieser Sicht der Preis, der für die Verminderung von Gesundheitsrisiken durch Adipositas gesamtgesellschaftlich zu zahlen ist.

Übergewicht ist in allen Industrienationen weit verbreitet. Annähernd 7\% der Krankheitskosten in Deutschland sind der Adipositas zuzuschreiben - zirka 8 Milliarden Euro pro Jahr [Wing et al., 1994]. Adipositas ist über das Körpergewicht definiert. Weitgehende Übereinstimmung besteht hier in der Verwendung des Body Mass Index (BMI = Körpergewicht [kg] / Körperlänge $\left[\mathrm{m}^{2}\right]$ ) [Garrow, 1988; Deutsche Adipositas Gesellschaft, 1995]. Wir sprechen von Normalgewicht bei einem BMI von 18,5 bis 24,9, von Prä-Adipositas bei einem BMI von 25,0 bis 29,9, von Adipositas Grad I bei einem BMI von 30,0 bis 34,9, von Adipositas Grad II bei einem BMI von 35,0 bis 39,9 und Adipositas Grad III bei einem BMI $\geq 40,0$. Gesundheitsrisiken und erhöhte Mortalität [Calle et al., 1999] sind mit Adipositas besonders dann verbunden, wenn ein bestimmter Fettverteilungstyp vorliegt. Die Folgerisiken sind besonders hoch bei der so genannten «Stammfettsucht», auch androide oder viszerale Adipositas genannt, bei der die Fettvermehrung besonders im Abdominalbereich besteht. Zirka $80 \%$ der Männer, aber nur 15\% der Frauen weisen diese Fett- verteilung auf. Häufiger bei Frauen ist der gynoide, gluteal-femorale Fettverteilungstyp, der bei $85 \%$ der Frauen und nur bei zirka 20\% der Männer beobachtet wird.

Adipositas geht nicht nur mit erhöhten Gesundheits- und Mortalitätsrisiken einher, sondern auch mit Vorurteilen und Diskriminierung. Schlankheit wird fälschlicherweise oft gleichgesetzt mit Schönheit und Erfolg, Übergewicht hingegen mit Trägheit, mangelnder Willensstärke, Dummheit und Unattraktivität [Klesges et al., 1990]. Was kann man tun, um Gewicht abzunehmen, die Risiken von Übergewicht zu mindern und von anderen mehr akzeptiert zu werden? Übersichten über Gewichtsabnahmeprogramme zeigen, dass gewichtsreduzierende Diäten durchaus effektiv sind und die Betroffenen im Verlauf der Diät abnehmen [Wadden, 1993]. Allerdings erreichen die wenigsten, die sich einer Diät oder einem gewichtsreduzierenden Programm unterziehen, Normalgewicht. Noch relevanter aber ist, das sie ihr Gewicht nicht auf Dauer reduzieren. Jeffery et al. [2000, p. 13] resümiert: "Overweight people readily initiate weight control efforts and with professional assistance, are quite able to persist, and lose weight for several months. They also experience positive outcomes in medical, psychological and social domains ... Nevertheless, they almost always fail to maintain the behaviour changes that brought them these positive results". Diätprogramme scheinen nicht nur häufig nutzlos zu sein, als Folge von Diäten wird ein so genannter «Jojo-Effekt» diskutiert, so dass Diäten langfristig sogar zu einer Gewichtzunahme führen können [Brownell und Rodin, 1994]. Diätverhalten kann auch bulimische oder anorektische Essstörungen auslösen [Killen et al., 1996].

Das vorliegende Schwerpunktheft befasst sich mit Entstehungsbedingungen und Therapiemöglichkeiten bei Adipositas. Während Zeitpunkt und Größe der jeweilig nächsten Mahlzeit nahezu vollständig vom freiem Willen bestimmt werden können, hängt die Gesamtmenge der über einen längeren Zeitraum aufgenommenen Nahrungskalorien stark von einem Regulationsmechanismus ab, dessen phylogenetische Aufgabe darin besteht, die verfügbare Energie im Organismus konstant zu halten. Jeder Versuch, Adipositas zu behandeln, ist ein Eingriff in diesen Mechanismus. Das rasch wachsende Wissen über die Prozesse der Gewichtsregulation, wie sie in 
dem einleitenden Beitrag von Cuntz [2002] dargestellt werden, ist die Grundlage, auf der die Möglichkeiten und Ansatzpunkte der Adipositastherapie fußen.

Da Körpergröße und Gewicht wohl die am einfachsten zu erhebenden anthropometrischen Daten sind, ist die epidemiologische Literatur zur Adipositas, die von Munsch [2002] zusammengefasst wird, mittlerweile extrem umfangreich und erlaubt wertvolle Rückschlüsse auf die Entstehung und die Folgen des Übergewichts. Dabei sind Adipöse als Gruppe weder bezüglich ihrer genetischen Ausstattung noch bezüglich des sozialen Status, psychologischer Auffälligkeiten oder auch nur des Essverhaltens eine homogene Gruppe. Als gesonderte Subgruppe haben die Adipösen, die anfallsartig große Nahrungsmengen essen («Binge Eating»), die besondere Aufmerksamkeit der Wissenschaftler erregt, da sich in dieser Gruppe besonders viele mit psychiatrischer Komorbidität finden. Das Phänomen des Binge Eating wird im Beitrag von de Zwaan [2002] dargestellt.

Die Häufigkeit der Adipositas nimmt besonders bedrohlich bei Kindern und Jugendlichen zu. Da anscheinend die Erfolge der Adipositastherapie in jüngeren Altersgruppen günstiger sind als im Erwachsenenalter, wird zunehmend Wert auf die frühe Behandlung und Prävention der Adipositas gelegt. In einer kontrollierten Studie haben Lehrke und Laessle [2002] untersucht, ob die Einbeziehung der Eltern in Therapieprogramme mit Kindern und Jugendlichen von Vorteil ist.

Eine Übersicht über die Geschichte, die Techniken und die Effizienz der Verhaltenstherapie bei Adipositas gibt der Beitrag von Benecke [2002]. Verhaltenstherapie muss sich dabei auch mit den symptomnahen Bereichen der Nahrungszubereitung und der Essensaufnahme auseinander setzen, wie sie in dem Beitrag von Wilhelm und Kollegen [2002] für die stationäre Therapie beschrieben werden.

Eine Therapieoption mit zunehmender Bedeutung sind die verschiedenen Techniken der operativen Magenverkleinerung, die sehr große und gut belegte Erfolge vorweisen können. Mit den psychischen Konsequenzen dieser solchermaßen «erzwungenen» Gewichtsreduktion beschäftigt sich der Beitrag von Kielmann und Herpertz [2002].

Schließlich ist die Aufgabe aller therapeutischen Anstrengungen die langfristige Gewichtsreduktion. Bisher sind die Erfolge aller diätetischen, aber auch der verhaltenstherapeutischen Maßnahmen enttäuschend. Der abschließende Beitrag von Leibbrand [2002] setzt sich mit der Frage auseinander, welche Bedingungen für einen positiven Verlauf gegeben sein müssen - ein Forschungsgebiet, das in Zukunft mit einem differentialtherapeutischen Ansatz ermöglichen könnte, durch die für jeden Betroffenen optimalen Therapiestrategien höhere Effizienz zu erreichen.

Die Zukunft der Adipositastherapie wird nicht in der Erfindung völlig neuer therapeutischer Techniken liegen, sondern in der systematischen Nutzung des vorhandenen immensen Wissens über die physiologische und psychische Regulation der Nahrungsaufnahme und ihrer Pathologie, in der besseren Charakterisierung der einzelnen Subgruppen von Übergewichtigen und in der Optimierung der Zuweisungskriterien zu den unterschiedlichen Therapieoptionen.

Manfred M. Fichter und U. Cuntz, Prien

\section{Literatur}

Benecke A: Verhaltenstherapie bei Adipositas. Verhaltenstherapie 2002;12:297-309.

Brown OJ, Konner MJ: An antrophological perspective on obesity. Paper presented at the conference on human obesity. The New York Academy of Science, 1987.

Brownell KD, Rodin J: The dieting maelstrom: Is it possible and advisable to lose weight? Am Psychol 1994:49:781-791.

Calle EE, Thun MJ, Petrelli JM, Rodriguez C, Heath CW Jr (1999): Body-mass index and mortality in a prospective cohort of U.S. adults. N Engl J Med 1999;341:1097-1105.

Cuntz U: Das homöostatische Prinzip und der «freie Wille» des Menschen - Biologische und psychologische Grundlagen der Adipositasbehandlung. Verhaltenstherapie 2002;12:268-277.

De Zwaan M: Binge eating disorder (BED) und Adipositas. Verhaltenstherapie 2002;12:288-295.
Deutsche Adipositas Gesellschaft: Richtlinien zur Therapie der Adipositas. Adipositas 1995;5:6-10.

Garrow JS: Obesity and Related Diseases. Edinburgh, Churchill Livingstone, 1988, pp 329-336.

Jefferey RW, Drewnowski A, Epstein LH, Stunkard AJ, Wilson GT, Wing RR, Hill DR: Long-term maintenance of weight loss: Current status. Health Psychol 2000;19:5-16.

Kielmann R, Herpertz S: Möglichkeiten und Grenzen der Adipositas-Chirurgie aus psychotherapeutischer Sicht. Verhaltenstherapie 2002;12:319-326.

Killen JD, Taylor B, Hayward C, Haydel KF, Wilson DW, Hammer L, Kraemer H, Blair-Greiner A, Strachowski D: Weight concerns influence the development of eating disorders: A 4-year prospective study. J Consult Clin Psychol 1996;64:936-940.

Klesges RV, Klem ML, Hanson CL, Eck LH, Ernst J, O'Laughlin D, Garrott A, Rife R: The effects of applicant's health status and qualifications on simulated hiring decisions. Int J Obes 1990;14:525-535.
Lehrke S, Laessle RG: Multimodale Therapie bei übergewichtigen Kindern: Therapieeffekte zu psychosozialen Variablen. Verhaltenstherapie 2002;12: 256-266.

Leibbrand R: Adipositas: Verlauf und verlaufsbeeinflussende Faktoren. Verhaltenstherapie 2002;12:327333.

Munsch S: Epidemiologie der Adipositas. Verhaltenstherapie 2002;12:278-287.

Wadden TA: The treatment of obesity: An overview; in Stunkard JA, Wadden TA (eds): Obesity: Theory and Therapy. New York, Raven, 1993, pp 197-218.

Wilhelm M, Strütt-Neeb P, Opielka C, Cuntz U: Verhaltenstherapeutische Ernährungstherapie für Adipositaspatienten. Verhaltenstherapie 2002;12:311-318. Wing RR, Blair E, Marcus M, Epstein LH, Harvey J: Year-long weight loss treatment of obese patients with type II diabetes: Does including an intermittent verylow-calorie diet improve outcome? Am J Med 1994;97: 354-362. 